\title{
Session III: Identifying and Defining Hazards and Potential Consequences II
}

\author{
Jeremy SWEET* \\ Sweet Environmental Consultants, 6 The Green, Willingham, Cambridge CB4 5JA, UK
}

PRESENTERS

Predictive modeling of the Consequences of hybridisation based on Fitness Estimates: the cases for lettuce and oilseed rape

Danny Hoofman

University of Amsterdam, The Netherlands

Weediness and Invasiveness of Transgenic Plants: Evaluating Screening Models and Their Predictions

Peter Caley

National Centre for Epidemiology and Population Health, Australian National University Canberra, Australia

Assessing the Potential Invasiveness of Pest Resistant Transgenic Plants

Rosie Hails

Centre for Ecology and Hydrology, Oxford Oxford, UK

This session examined how effects of GM plants can be distinguished from those of non-GM comparators, how these differences can be measured, and how effects on non-targets and on plant fitness can be assessed. This review focuses on issues concerning plant fitness and invasiveness, and explores how the consequences of changes can be evaluated in terms of their environmental impact for risk assessments.

All three authors discussed the first task, which is to determine which life cycle parameters and characteristics of a plant (e.g. fecundity, establishment, seedling survival, etc.) are most associated with fitness and therefore likely to increase fitness if they are modified. The second task is to try to determine whether increased fitness will lead to enhanced invasiveness.

The authors used background knowledge of the behavior and life cycle of conventional plants to determine the factors affecting their behavior and fitness. They conducted tests to determine the significance of life cycle parameters, and then used these parameters as primary inputs into models to estimate changes in fitness in recipient plants.

They discussed the development of plant (generic) and ecosystem models (e.g. matrix models), in order to predict changes in fitness and environmental conse-

*Corresponding author: jeremysweet303@aol.com quences. They assumed stochasticity in studied environments, both demographic and environmental.

Based on this knowledge and an understanding of existing and proposed niches for these plants, predictions of future behavior of introduced and GM plants in novel environments can be assessed. This will be useful for predicting:

- Impacts on populations

- Transgene frequency

- Impacts on specific ecosystems

- Cumulative effects

- Long term effects

- Large scale/landscape effects.

However, in order to develop more robust models, more data is often needed on the fundamental ecology of exposed sites, so that interactions with critical points in the life cycle can be studied (e.g. through elasticity analysis). In addition, more testing and verification of models is required in order determine their environmental sensitivity and to improve their "accuracy". Peter Caley argued that there are limitations to the predictions that can be made from a limited number of experiments, and that using screening models can also be problematic. He discussed whether controlled or phased experimental release is a way forward especially for perennial GM plant species. 
Rosie Hails and Danny Hoofman cautioned that fitness can be very environmentally sensitive and thus models are only as good as the environmental parameters included in them.

The challenge is to answer the key end point questions: "is the GM plant significantly more fit or invasive?" and "will the GM plant have an adverse environmental impact in one or more exposed ecosystems ?" This session showed the potential for models to help achieve these end points by improving the probability that prediction of an impact is correct. However the levels of uncertainty are still high in many cases. 